\title{
Intra-renal and urinary RNA expression of podcyte-associated molecules in patients with IgA nephropathy
}

\author{
Gang Wang ${ }^{1,3}$, Bonnie Ching Kwan ${ }^{1}$, Fernand Mac-Moune Lai ${ }^{2}$, Paul Cheung-Lung Choi ${ }^{2}$, Kai-Ming \\ Chow $^{1}$, Philip Kam-Tao Li ${ }^{1}$, Cheuk-Chun Szeto ${ }^{1}$

\footnotetext{
${ }^{1}$ Department of Medicine \& Therapeutics, Faculty of Medicine, The Chinese University of Hong Kong, Hong Kong, China;

${ }^{2}$ Department of Anatomical \& Cellular Pathology, Prince of Wales Hospital, The Chinese University of Hong Kong, Hong Kong, China;

${ }^{3}$ Division of Nephrology, Renmin Hospital of Wuhan University, Wuhan, China.

Email: ccszeto@cuhk.edu.hk
}

Received 14 April 2010; revised 7 May 2010; accepted 12 May 2010.

\begin{abstract}
Background Podocyte injury probably plays important roles in the pathogenesis of $\operatorname{IgA}$ nephropathy (IgAN). We studied intra-renal and urinary messenger RNA (mRNA) expression of podocyte-associated molecules in patients with IgAN. Methods We studied 43 consecutive patients with biopsy-proven IgAN. Intra-renal and urinary expression of mRNAs was determined and compared to that of 20 patients with nephrectomy for kidney cancer and 12 normal subjects. Results Intra-renal mRNA expression levels of nephrin, podocin and synaptopodin were significantly lower in patients with $\operatorname{IgAN}$ than that of controls. In contrast, their urinary mRNA expression levels were similar. Intra-renal gene expression of nephrin inversely correlated with proteinuria $(r=$ $-0.620, P<0.001)$, GFR $(r=0.538, P<0.001)$, and the degree of tubulointerstitial scarring $(\mathrm{r}=-\mathbf{0 . 4 2 3}, \mathrm{P}=$ 0.013). After followed for an average of $33.4 \pm \mathbf{1 2 . 6}$ months, intra-renal nephrin expression significantly correlated with the rate of GFR decline $(r=0.324, P$ $=0.041)$. Conclusions Intra-renal mRNA expression of podocyte associated molecules was down-regulated in patients with $\operatorname{IgAN}$, and the degree of downregulation of nephrin correlated with disease severity and the rate of progression. Our result supports the hypothesis that podocyte injury is an important component in the pathophysiology of IgAN.
\end{abstract}

Keywords: Glomerulonephritis; Proteinuria; Chronic Kidney Diseases

\section{INTRODUCTION}

Immunoglobulin A nephropathy (IgAN) is the most common form of primary glomerulonephritis throughout the world, and a good portion of patients with biopsy-proven
IgAN progresses to ESRD in 10 to 20 years [1-3].

The most prominent pathological changes of IgAN are deposition of pathogenic polymeric IgA1 in the mesangium, proliferation of mesangial cells, excessive synthesis of extracellular matrix (ECM), and infiltration of inflammatory cells. However, podocyte loss is a concomitant of increasing disease severity in IgAN [4]; podocyte loss may either cause or contribute to the progressive proteinuria, glomerular sclerosis and filtration failure in IgAN [4]. Podocyte injury might provide additional prognostic information in patients with $\operatorname{IgAN}$ [5]. More recently, it has been shown that Bcl-2 expression by podocytes exerts modulatory effects on cellular processes that contribute to progressive glomerular injury of IgAN [6], while humoral factors released from mesangial cells may alter glomerular permeability by reducing the expression of podocyte markers [7]. In the present study, we explore the intra-renal and urinary messenger RNA (mRNA) expression of podocyte-associated molecules in patients with $\operatorname{IgA}$ nephropathy.

\section{PATIENTS AND METHODS}

\subsection{Subjects}

We studied 43 consecutive patients with IgA nephropathy confirmed by kidney biopsy in the Prince of Wales Hospital, Hong Kong, between 2004 and 2007. Patients with other coexisting renal pathology, and recurrent IgA nephropathy after kidney transplantation, were excluded. The study was approved by the Clinical Research Ethical Committee of the Chinese University of Hong Kong, all patients provided informed consent. Clinical data including serum creatinine and 24 hours urine protein were recorded at the time of kidney biopsy. Glomerular filtration rate (GFR) was estimated by a standard equation [8]. We studied normal renal tissue from the nephrectomy 
specimen of 20 patients with renal cell carcinoma (all clear cell type) and urine sediment from 12 healthy subjects as controls for intra-renal and urinary mRNA expression study, respectively.

After renal biopsy, all patients were followed every 2 months for at least 12 months. Renal function and the degree of proteinuria were assessed at every visit. Disease progression was measured by the rate of GFR decline, which was calculated by the least-square regression method [9]. Treatment for individual patient was determined by the responsible physician and not affected by this study. All physicians were blinded from the results of mRNA expression.

\subsection{Sample Preparation}

Immediately after kidney biopsy, the renal tissue was fixed in $10 \%$ neutral buffered formaldehyde overnight and then dehydrated by alcohol and embedded in paraffin for intra-renal mRNA expression. Ten $10 \mu \mathrm{m}$ sections were cut from the formalin-fixed and paraffin-embedded (FFPE) tissue blocks using a microtome and pooled in a $1.5 \mathrm{ml}$ microcentrifuge tube. The sections were then treated by xylene for 3 minutes at $50^{\circ} \mathrm{C}$ and washed by $100 \%$ ehthanol twice. Air dry the pellet for 30 minutes at room temperature.

Urine specimen was collected and processed immediately, or stored in $4{ }^{\circ} \mathrm{C}$ overnight. Urine sample was centrifuged at $3000 \mathrm{~g}$ for 30 minutes and at $13000 \mathrm{~g}$ for 5 minutes at $4^{\circ} \mathrm{C}$. Supernatant was discarded and the urinary cell pellet was lysed by RNA lysis buffer (Qiagen Inc, Ontario, Canada). Specimens were then stored at $-80^{\circ} \mathrm{C}$ until use.

\subsection{Measurement of MRNA Levels}

RecoverALL ${ }^{\text {TM }}$ total nucleic acid isolation kit and MirVana $^{\mathrm{TM}}$ miRNA isolation kit (Ambion, Inc. Austin, TX, USA) were used for the extraction of total RNA from FFPE tissue and urinary sediment according to the manufacturer's protocol.

High Capacity cDNA Reverse Transcription Kit (Applied Biosystems, Foster City, CA, USA) were used for reverse transcription. $10 \mu \mathrm{l}$ total RNA was mixed with 2 $\mu 1$ specific primers, $0.8 \mu 1100 \mathrm{mM}$ dNTPs (with dTTP), $2 \mu 110 \times$ reverse transcription buffer, $1 \mu 1$ (50U) MultiScribe ${ }^{\mathrm{TM}}$ Reverse Transcriptase, $1 \mu \mathrm{l}$ RNase inhibitor (20 U/ $\mu \mathrm{l})$ and made up to $20 \mu \mathrm{l}$ with $\mathrm{H}_{2} \mathrm{O}$. Reverse transcription was performed at $25^{\circ} \mathrm{C}$ for 10 minutes, $37^{\circ} \mathrm{C}$ for 120 minutes and $85^{\circ} \mathrm{C}$ for 5 minutes. The resulting cDNA was stored in $-80^{\circ} \mathrm{C}$ until use.

Intra-renal and urinary mRNA expression of nephrin, podocin and synaptopodin were quantified by RT-QPCR using the ABI Prism 7900 Sequence Detection System (Applied Biosystems, Foster City, CA, USA). Commer- cially available Taqman primers and probes, including 2 unlabeled PCR primers and 1 FAM $^{\mathrm{TM}}$ dye-labeled TaqMan ${ }^{\circledR}$ MGB probe were used for all the targets (all from Applied Biosystems). The primer and probe set was deliberately designed across the intron-exon boundary so as not to detect probable genomic DNA. For RT-QPCR, $10 \mu 1$ universal master mix, $1 \mu 1$ primer and probe set, $1.33 \mu \mathrm{l}$ cDNA and $7.67 \mu 1 \mathrm{H}_{2} \mathrm{O}$ were mixed to make a $20 \mu \mathrm{l}$ reaction volume. Each sample was run in triplicate. RT-QPCR were performed at $50^{\circ} \mathrm{C}$ for $2 \mathrm{~min}$ utes, $95^{\circ} \mathrm{C}$ for 10 minutes, followed by 40 cycles at $95^{\circ} \mathrm{C}$ for 15 seconds and $60^{\circ} \mathrm{C}$ for 1 minute. $\beta$-Glucuronidase (GUSB, Applied Biosystems) was used as housekeeping genes to normalize the mRNA expression [10]. Results were analyzed with Sequence Detection Software version 2.0 (Applied Biosystems). In order to calculate the differences of expression level for each target among samples, the $\Delta \Delta \mathrm{C}_{\mathrm{T}}$ method for relative quantitation was used. Average expression level of normal renal tissue from patients with kidney clear cell cancer and urinary sediment from healthy controls was used as calibrator for intra-renal expression and the expression level of targets was a ratio relative to that of the controls.

\subsection{Assessment of Renal Scarring}

Analysis of renal fibrosis was determined on $4 \mu \mathrm{m}$ paraffin-embedded sections stained by Periodic Acid Schiff (PAS) or Jones silver stain. The severity of renal fibrosis was scored by an experienced pathologist who was blinded to the results of molecular studies. The severity of glomerulosclerosis was represented by the percentage of sclerotic glomeruli in total glomeruli obtained from biopsy. For tubulointerstitial scarring, ten microscopic fields were viewed at magnification of $200 \times$ and scored subjectively from 0 to $100 \%$ for each patients. The severity of tubulointerstitial scarring was represented by the mean of ten scores.

\subsection{Statistical Analysis}

Statistical analysis was performed by SPSS for Windows software version 13.0 (SPSS Inc., Chicago, IL). All the results were presented in mean \pm SD for data normally distributed and median (lower and upper quartiles) for the others. Since data of gene expression levels were highly skewed, either log transformation or nonparametric statistical methods were used. We used MannWhitney $U$ test to compare gene expression levels between groups and Spearman's rank-order correlations to test associations between gene expression levels and clinical parameters. When no detectable level of a transcript was found (defined as no detectable level after 40 cycles of RT-QPCR) and there was zero value, a value equal to half of the minimum observed gene expression 
level was assigned. A P value of below 0.05 was considered statistically significant. All probabilities were twotailed.

\section{RESULTS}

The demographic and baseline clinical data of the study subjects were summarized in Table 1 . As compared to controls, patients with IgA nephropathy had significantly higher level of proteinuria and worse renal function. Histological studies showed that the percentage of glomerulosclerosis and tubulointerstitial scarring of patients were $28.33 \pm 26.97 \%$ and $25.60 \pm 24.06 \%$ respectively.

\subsection{Levels of Gene Expression}

The intra-renal and urinary expression of various mRNA species are compared and summarized in Figure 1. As compared to controls, patients with IgA nephropathy had lower intra-renal gene expression of nephrin $(0.16$ [0.07 -0.39 ] versus 1.02 [0.81-1.43], $\mathrm{P}<0.001)$, podocin $(0.49$ [0.12-1.47] versus 0.95 [0.66-1.81], $\mathrm{P}=0.019)$ and synaptopodin (0.60 [0.31-0.83] versus 0.92 [0.72-1.30], $\mathrm{P}<$ $0.001)$. Urinary mRNA expression of nephrin and synaptopodin, on the contrary, was marginally lower in patients with IgA nephropathy than controls $(0.16$ [0.06$0.67]$ versus 0.80 [0.27-1.18], $\mathrm{P}=0.091$ for nephrin, and 0.31 [0.14-0.85] versus 0.94 [0.30-2.94], $\mathrm{P}=0.051$ for synaptopodin). In contrast, their urinary expression of podocin was similar $(0.21$ [0.06-0.64] versus 0.40 [0.00 -1.64], $\mathrm{P}=0.894)$. There was no significant correlation between intra-renal and urinary mRNA expression of studied molecules (details not shown).

\subsection{Relation with Clinical Data}

Since intra-renal gene expression of nephrin, podocin and synaptopocin was significantly different between patients and controls, we further explored the relation between their level of intra-renal expression and clinical parameters. The result was summarized in Figure 2. In short, proteinuria inversely correlated with intra-renal gene expression of nephrin $(\mathrm{r}=-0.620, \mathrm{P}<0.001)$ and synaptopodin $(\mathrm{r}=-0.389, \mathrm{P}=0.003)$, but not podocin $(\mathrm{r}$ $=-0.237, \mathrm{P}=0.073$ ) (Figure 2(a)). GFR significantly correlated with intra-renal gene expression of nephrin $(\mathrm{r}$ $=0.538, \mathrm{P}<0.001)$, but not podocin $(\mathrm{r}=0.205, \mathrm{P}=$ $0.123)$ or synaptopodin $(\mathrm{r}=0.193, \mathrm{P}=0.147)$ (Figure 2(b)).

\subsection{Relation with Histological Damage}

Relations between intra-renal gene expression of nephrin, podocin and synaptopodin were summarized in Figure 3. Briefly, tubulointerstitial scarring significantly correlated with intra-renal gene expression of nephrin $(\mathrm{r}=-0.423$, $\mathrm{P}=0.013)$, but not podocin $(\mathrm{r}=-0.171, \mathrm{P}=0.333)$ or synaptopodin $(r=-0.136, P=0.445)$. Glomerular scarring, however, did not significantly correlate with any of the targets studied (details not shown).

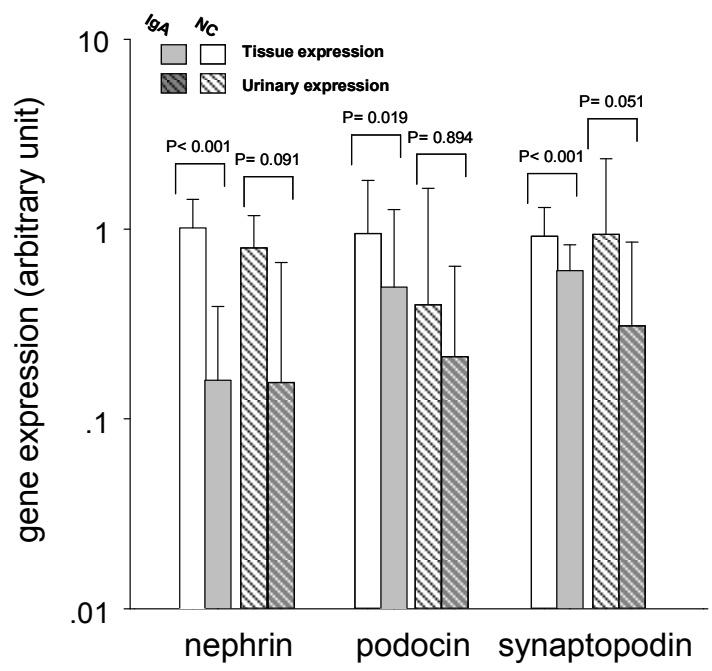

Figure 1. Comparison of intra-renal and urinary expression of mRNAs between patients with IgA nephropathy and controls. Data are compared by Mann-Whitney U test. Levels are represented as ratio to the average of controls.

Table 1. Demographic and baseline clinical data of the subjects.

\begin{tabular}{lccc}
\hline & IgAN & Biopsy control & Healthy subject \\
\hline No. of case & 43 & 20 & 12 \\
Sex (M:F) & $27: 16$ & $13: 7$ & $7: 5$ \\
Age (year) & $48.37 \pm 12.64$ & $52.20 \pm 8.30$ & $31.33 \pm 3.87$ \\
Proteinuria (g/day) & $1.13 \pm 1.13$ & $0.01 \pm 0.03$ & 0.00 \\
$\begin{array}{l}\text { Serum creatinine } \\
(\mu \mathrm{mol} / \mathrm{l})\end{array}$ & $175.07 \pm 123.03$ & $95.06 \pm 23.02$ & -- \\
$\begin{array}{l}\text { GFR } \\
\left(\mathrm{ml} / \mathrm{min} / 1.73 \mathrm{~m}^{2}\right)\end{array}$ & $50.44 \pm 29.50$ & $76.09 \pm 26.02$ & -- \\
\hline IgAN, immunoglobulin A nephropathy; GFR, glomerular filtration rate. &
\end{tabular}



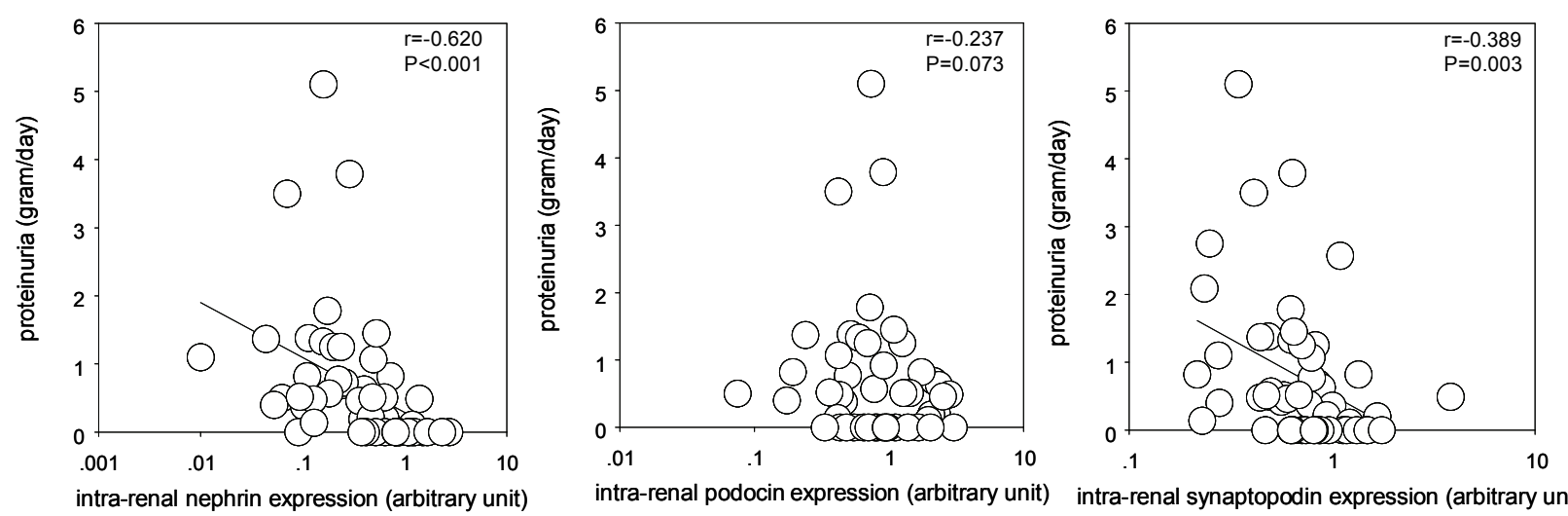

(a)
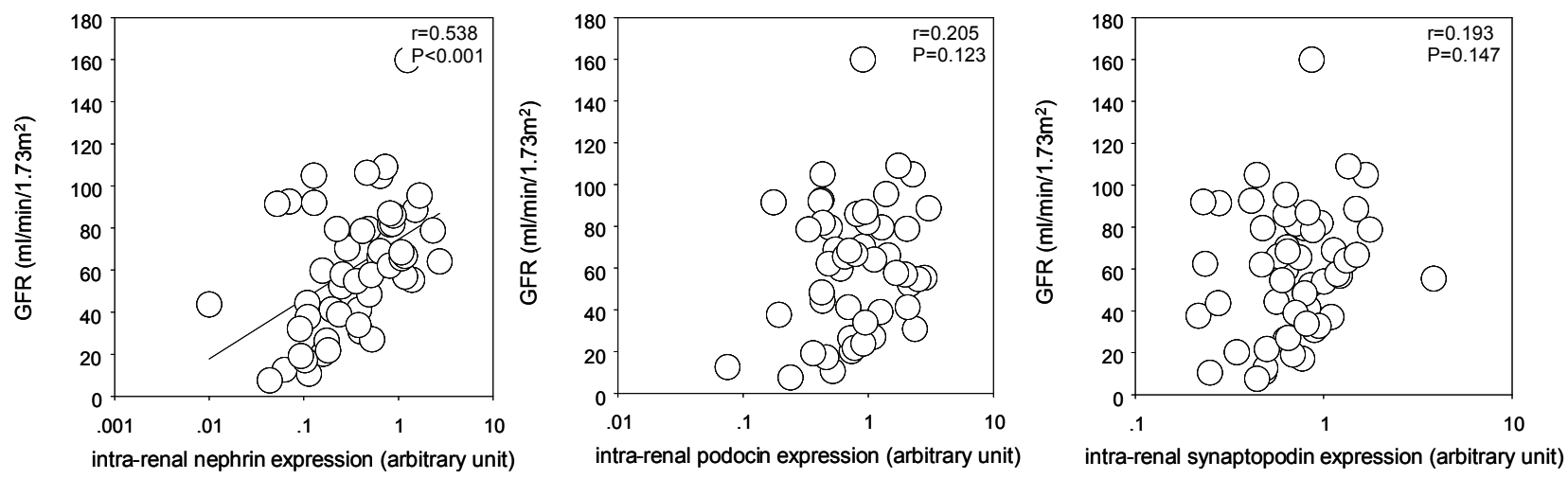

(b)

Figure 2. Relation between intra-renal expression of mRNAs and (a) proteinuria and (b) glomerular filtration rate (GFR). Data are compared by Spearman's rank correlation coefficient.
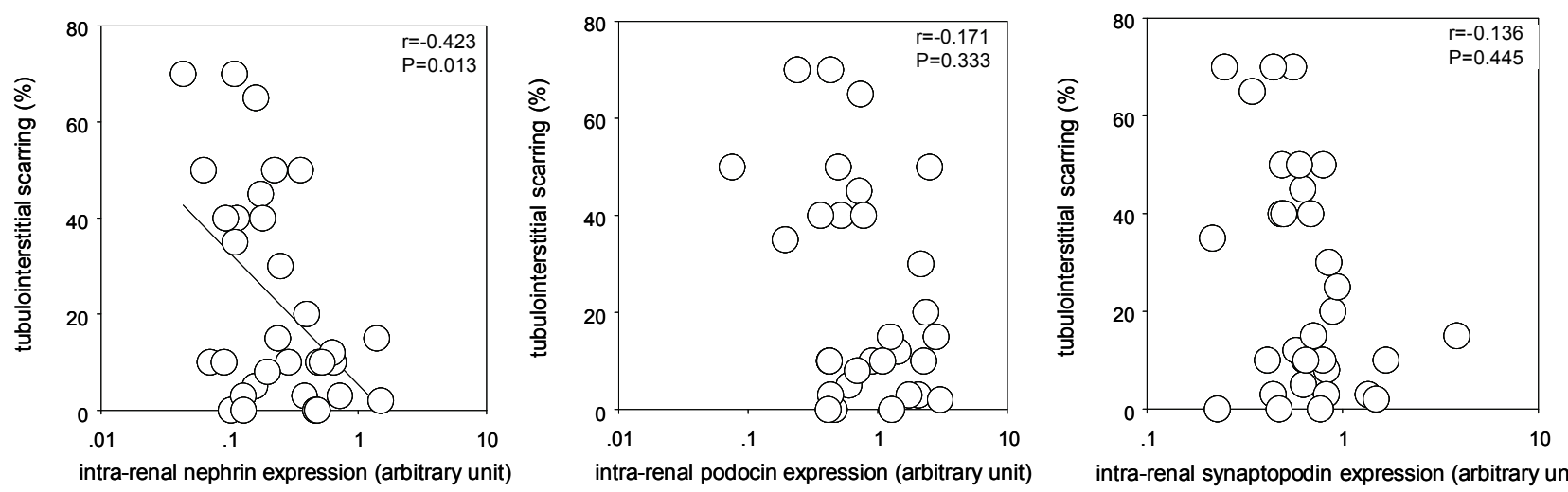

Figure 3. Relation between intra-renal expression of mRNAs and tubulointerstitial scarring. Data are compared by Spearman's rank correlation coefficient.

\subsection{Relation with Renal Function Decline}

The average duration of follow up was $33.4 \pm 12.6$ months; the average rate of GFR decline was $-0.24 \pm$ $0.62 \mathrm{ml} / \mathrm{min} / \mathrm{month}$. The rate of GFR decline significantly correlated with intra-renal gene expression of nephrin $(\mathrm{r}=0.324, \mathrm{P}=0.041)$ (Figure 4), indicating that the lower the intra-renal gene expression of nephrin, the faster the renal function decline. The rate of GFR decline did not significantly correlate with podocin $(\mathrm{r}=0.177, \mathrm{P}$ $=0.274)$ or synaptopodin $(\mathrm{r}=0.224, \mathrm{P}=0.164)$ (Figure $4)$.

\section{DISCUSSION}

In the present study, we found intra-renal expression of 

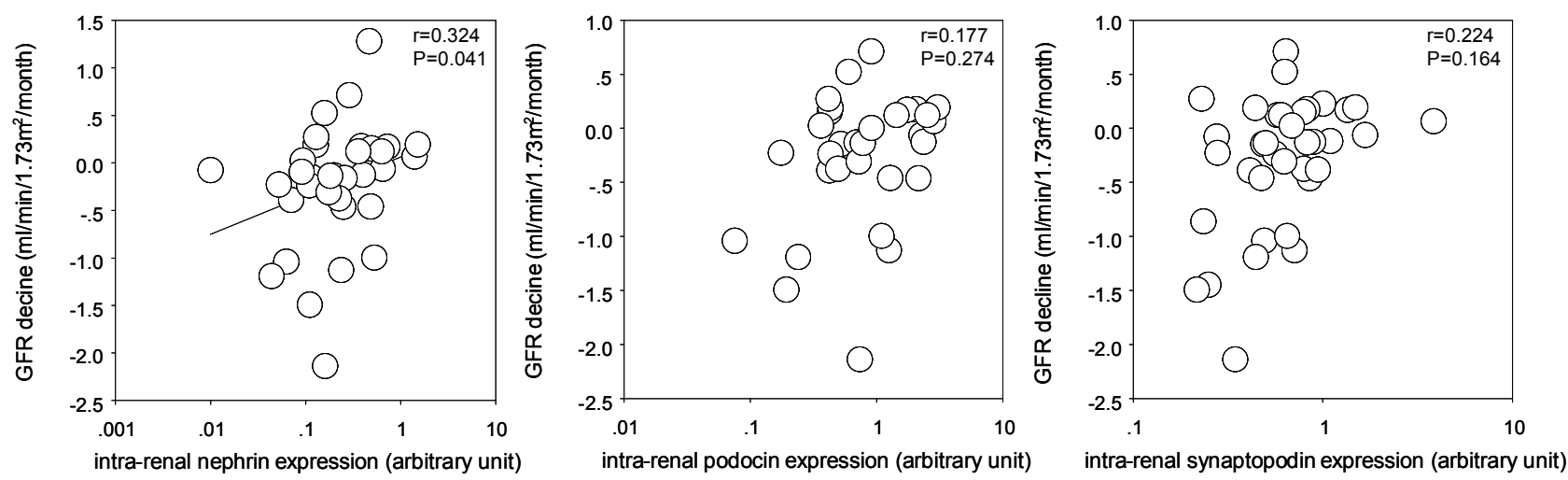

Figure 4. Relation between intra-renal expression of mRNAs and the rate of change in estimated glomerular filtration rate (GFR). Data are compared by Spearman's rank correlation coefficient. Negative change in GFR indicates worsening of renal function.

podocyte-associated molecules, nephrin, podocin and synaptopodin, was down-regulated in patients with IgAN while urinary expression of these molecules was unchanged. We also found intra-renal expression of these molecules, especially nephrin, significantly correlates with disease severity and progression of IgAN.

Podocyte injury in IgAN has been attributed to direct or indirect effects of serum IgA1 and mesangial-derived cytokines such as TNF- $\alpha$ or TGF- $\beta[7,11]$. Shedding of podocytes from glomerulus to urine and down-regulation of nephrin, a crucial component of the slit diaphragm, has been proved in patients with IgAN [12-15]. In line with these previous results, we found that besides nephrin, scaffolding and cytoskeleton related molecules podocin and synaptopodin were also down-regulated in patients with $\operatorname{IgAN}$, as also proved by a recent in vitro study [7]. Though the exact mechanism by which these molecules are down-regulated has not been examined, these results suggests that there might be pervasive down-regulation of podocyte-associated molecules including slit diaphram components, scaffolding and cytoskeleton related molecules in IgAN.

We have previously reported that urinary gene expression of podocyte-associated molecules in patients with other kidney diseases such as DN and HTN is significantly higher than normal controls [16,17], suggesting podocytes detach into the urine in these conditions. Indeed, more viable podocytes are proved to shed from the glomerulus in patients with glomerular diseases than healthy controls [18]. On the contrary, urinary gene expression of podocyte-associated molecules tends to be lower in patients with IgAN than that in normal subjects in the present study. This result suggests that podocyte loss in IgAN for a different mechanism (for example, apoptosis).

In the present study, the intra-renal expression of nephrin, but not podocin or synaptopodin, was found significantly correlated with disease severity and pro- gression of IgAN. The explanation for this discrepancy among these podocyte-associated molecules remains unclear. Of note, nephrin has been proved to be an indispensable component of slit diaphragm and loss of nephrin is related to increased permeability of glomerular filtration barrier which may lead to proteinuria and disease progression $[19,20]$. Further, nephrin is a signaling adhesion molecule and link to the actin skeleton of podocyte [20]. Loss of nephrin may therefore facilitate unhinging adjacent foot processes through the dysfunction of the actin skeleton system. Taken together, our results suggest that nephrin loss may play a pivotal role in the impairment of slit diaphragm function.

There are a number of inadequacies of our study. First, we used normal renal tissue from the nephrectomy specimen of patients with renal cell carcinoma as control. However, the renal function of these patients was not normal (average GFR less than $80 \mathrm{ml} / \mathrm{min}$ ), and intra-renal expression of podocyte-associated molecules might be affected by the renal insufficiency. In theory, renal tissue from subjects with normal renal function (for example, kidney donors) could be used as controls.

Secondly, we did not enumerate podocyte number in this study. It was likely that the change in intra-renal expression of podocyte-associated molecules is a result of decreasing podocyte number, but it was also possible that there was down-regulation of podocyte associated molecules per cell. Furthermore, this study is only cross-sectional, and it is possible that the mRNA expression of podocyte-associated molecules changes with disease progression and therapy. Future studies are needed to investigate the serial change in expression of these targets as the disease progresses or following various therapy strategies.

In summary, we show that intra-renal gene expression of podocyte-associated molecules, especially nephrin, is down-regulated in patients with IgAN, and the degree of down-regulation of nephrin correlates with disease se- 
verity and rate of progression. Our result supports the hypothesis that podocyte injury is an important component in the pathophysiology of IgAN.

\section{ACKNOWLEDGEMENTS}

This study was supported in part by the CUHK research accounts 6901031 and 7101215 . The results presented in this paper have not been published previously in whole or part, except in abstract format. All authors declare no conflict of interest.

\section{REFERENCES}

[1] Donadio, J.V. and Grande, J.P. (2002) IgA nephropathy. New England Journal of Medicine, 347(10), 738-748.

[2] Berthoux, F.C., Mohey, H. and Afiani, A. (2008) Natural history of primary IgA nephropathy. Seminars in $\mathrm{Ne}$ phrology, 28(1), 4-9.

[3] Tan, C.H., Loh, P.T., Yang, W.S., Chan, C.M. (2008) Mycophenolate mofetil in the treatment of IgA nephropathy: A systematic review. Singapore Medical Journal, 49(10), 780-785.

[4] Lemley, K.V., Lafayette, R.A., Safai, M., Derby, G., Blouch, K., Squarer, A. and Myers, B.D. (2002) Podocytopenia and disease severity in IgA nephropathy. Kidney International, 61(4), 1475-1485.

[5] Hishiki, T., Shirato, I., Takahashi, Y., Funabiki, K., Horikoshi, S., Tomino, Y. (2001) Podocyte injury predicts prognosis in patients with IgA nephropathy using a small amount of renal biopsy tissue. Kidney and Blood Pressure Research, 24(2), 99-104.

[6] Qiu, L.Q., Sinniah, R. and I-Hong Hsu, S. (2004) Downregulation of Bcl-2 by podocytes is associated with progressive glomerular injury and clinical indices of poor renal prognosis in human IgA nephropathy. Journal of the American Society of Nephrology, 15(1), 79-90.

[7] Lai, K.N., Leung, J.C., Chan, L.Y., Saleem, M.A., Mathieson, P.W., Tam, K.Y., Xiao, J., Lai, F.M. and Tang, S.C. (2009) Podocyte injury induced by mesangial-derived cytokines in IgA nephropathy. Nephrology Dialysis Transplantation, 24(1), 62-72.

[8] Levey, A.S., Bosch, J.P., Lewis, J.B., Greene, T., Rogers, N. and Roth, D. (1999) A more accurate method to estimate glomerular filtration rate from serum creatinine: A new prediction equation. Modification of Diet in Renal Disease Study Group. Annals of Internal Medicine, 130(6), 461-470.

[9] Mitch, W. (1986) Measuring the rate of progression of renal insufficiency. In: Mitch, W., Stein, J. and Brenner, B., Eds., The Progressive Nature of Renal Disease, Churchill Livingstone Inc, New York, 203-220.
[10] Bhandari, S., Watson, N., Long, E., Sharpe, S., Zhong, W., Xu, S.Z. and Atkin, S.L. (2008) Expression of somatostatin and somatostatin receptor subtypes 1-5 in human normal and diseased kidney. Journal of Histochemistry \& Cytochemistry, 56(8), 733-743.

[11] Wang, C., Peng, H., Tang, H., Liu, X., Chen, Z., Lou, T. and $\mathrm{Yu}, \mathrm{X}$. (2007) Serum IgA1 from IgA nephropathy patients induces apoptosis in podocytes through direct and indirect pathways. Clinical and Investigative Medicine, 30(6), E240-249.

[12] Hara, M., Yanagihara, T. and Kihara, I. (2007) Cumulative excretion of urinary podocytes reflects disease progression in IgA nephropathy and Schonlein-Henoch purpura nephritis. Clinical Journal of the American Society of Nephrology, 2(2), 231-238.

[13] Ng, W.L., Chan, K.W., Yeung, C.K. and Kwan, S. (1984) Peripheral glomerular capillary wall lesions in IgA nephropathy and their implications. Pathology, 16(3), 324330.

[14] Gagliardini, E., Benigni, A., Tomasoni, S., Abbate, M., Kalluri, R. and Remuzzi, G. (2003) Targeted downregulation of extracellular nephrin in human IgA nephropathy. American Journal of Nephrology, 23(4), 277-286.

[15] Doublier, S., Ruotsalainen, V., Salvidio, G., Lupia, E., Biancone, L., Conaldi, P.G., Reponen, P., Tryggvason, K. and Camussi, G. (2001) Nephrin redistribution on podocytes is a potential mechanism for proteinuria in patients with primary acquired nephrotic syndrome. American Journal of Pathology, 158(5), 1723-1731.

[16] Wang, G., Lai, F.M., Lai, K.B., Chow, K.M., Li, K.T. and Szeto, C.C. (2007) Messenger RNA expression of podocyte-associated molecules in the urinary sediment of patients with diabetic nephropathy. Nephron Clinical Practice, 106(4), c169-c179.

[17] Wang, G., Lai, F.M., Kwan, B.C., Lai, K.B., Chow, K.M., Li, P.K. and Szeto, C.C. (2009) Podocyte loss in human hypertensive nephrosclerosis. American Journal of $\mathrm{Hy}$ pertension, 22(3), 300-306.

[18] Vogelmann, S.U., Nelson, W.J., Myers, B.D. and Lemley, K.V. (2003) Urinary excretion of viable podocytes in health and renal disease. American Journal of Physiology Renal Physiology, 285(1), F40-48.

[19] Ruotsalainen, V., Ljungberg, P., Wartiovaara, J., Lenkkeri, U., Kestila, M., Jalanko, H., Holmberg, C. and Tryggvason, K. (1999) Nephrin is specifically located at the slit diaphragm of glomerular podocytes. Proceedings of the National Academy of Sciences, USA, 96(14), 79627967.

[20] Jalanko, H. (2003) Pathogenesis of proteinuria: Lessons learned from nephrin and podocin. Pediatric Nephrology, 18(6), $487-491$. 